Western University

Scholarship@Western

Brain and Mind Institute Researchers'

Publications

Brain and Mind Institute

$5-1-2003$

\title{
Effects of ethanol on anti-saccade task performance.
}

Sarah A Khan

Kristen Ford

Brian Timney

Stefan Everling

Follow this and additional works at: https://ir.lib.uwo.ca/brainpub

Part of the Neurosciences Commons, and the Psychology Commons

Citation of this paper:

Khan, Sarah A; Ford, Kristen; Timney, Brian; and Everling, Stefan, "Effects of ethanol on anti-saccade task performance." (2003). Brain and Mind Institute Researchers' Publications. 6.

https://ir.lib.uwo.ca/brainpub/6 


\section{Sarah A. Khan · Kristen Ford • Brian Timney • Stefan Everling \\ Effects of ethanol on anti-saccade task performance}

Received: 2 July 2002 / Accepted: 10 January 2003 / Published online: 4 March 2003

(C) Springer-Verlag 2003

\begin{abstract}
It has been shown that saccade-related neurons in the superior colliculus (SC) display an increased level of prestimulus activity and a higher stimulus-related burst in action potentials preceding direction errors in the antisaccade task compared with correct anti-saccades. From this, it has been hypothesized that errors occur when the incoming visual signal in the SC passes a threshold and triggers a reflexive saccade. This hypothesis predicts that an attenuated visual signal will reduce the number of direction errors. Since ethanol has been shown to have a suppressive effect on cortical visual event-related potentials (ERPs), the purpose of the present study was to investigate the effects of moderate ethanol consumption on anti-saccade performance. Under both placebo and ethanol conditions, we recorded ERPs and measured eye movements in male subjects during the performance of an anti-saccade task in which the fixation point disappeared $200 \mathrm{~ms}$ prior to stimulus presentation. Compared with the placebo condition, we found in the ethanol condition: (1) a decrease in ERP amplitudes during the gap period and after stimulus presentation, (2) an increase in the latencies of anti-saccades, and (3) a decrease in the percentage of direction errors. These data demonstrate the effects of ethanol on anti-saccade task performance and provide further support for the hypothesis that errors in the antisaccade task are triggered by the incoming visual signal.
\end{abstract}

A preliminary report of the data presented in this report has appeared in abstract form for the Society for Neuroscience (Khan, Ford, Everling and Timney 2002).

S. A. Khan · K. Ford · B. Timney $\cdot$ S. Everling

Department of Psychology,

The University of Western Ontario,

Social Science Centre, London, Ontario, N6A 5C2, Canada

S. Everling (

Department of Physiology and Pharmacology,

The University of Western Ontario,

Social Science Centre, London, Ontario, N6A 5C2, Canada

e-mail: severlin@uwo.ca

Tel.: +1-519-6858500 ext 34359

Fax: +1-519-6613961
Keywords Eye movements - Evoked potentials . Alcohol · Inhibition

\section{Introduction}

The suppression of reflexive responses is often necessary to correctly execute a voluntary behaviour. A neuropsychological task extensively employed in recent years to test this ability is the anti-saccade task (Everling and Fischer 1998). In this task the subject is instructed to look away from a suddenly appearing peripheral stimulus to its mirror position in space (Hallett 1978; Hallett and Adams 1980). Successful performance requires the suppression of the phylogenetically old "visual grasp" reflex (Hess et al. 1946), an orienting eye movement towards a visual stimulus. This reflex is primarily mediated by the superior colliculus (SC) in the midbrain, the intermediate layers of which receive visual inputs (for review see Wurtz and Goldberg 1989).

The SC contains distinct groups of neurons with fixation or saccade-related activity. Fixation-related neurons are located in the rostral pole of the SC (Munoz and Wurtz 1992; Everling et al. 1998b), and are tonically active during fixation but pause in activity before saccades. On anti-saccade trials, these neurons increase their tonic activity, which is consistent with the idea of the occurrence of increased fixation on anti-saccade trials (Everling et al. 1999). There is some evidence from human lesion studies that this increased fixation activity may be mediated, at least in part, by direct and indirect frontal cortical projections to the SC, indicating a topdown control mechanism that prevents the SC from triggering a reflexive saccade. Patients with frontal cortex lesions have difficulties suppressing reflexive saccades in the anti-saccade task (Gaymard et al. 1998; Guitton et al. 1985; Pierrot-Deseilligny et al. 1993). The frontal eye fields (FEF), supplementary eye fields (SEF), and dorsolateral prefrontal cortex (DLPFC) are considered to be predominantly frontal areas involved in this top-down control (Guitton et al. 1985; Pierrot-Deseilligny et al. 
A

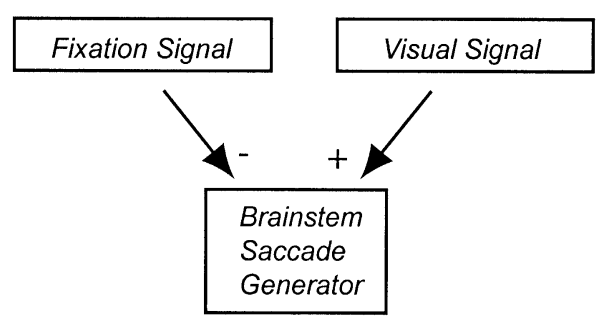

B
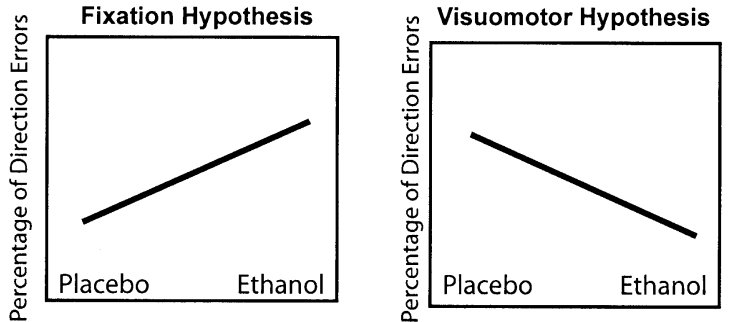

Fig. 1A,B Schematic representation of the fixation and visuomotor hypothesis (A), and predicted effects of ethanol on anti-saccade task performance (B). The fixation hypothesis predicts that ethanol will increase the number of direction errors (B, left diagram), whereas the visuomotor hypothesis predicts a decrease in the number of direction errors by ethanol in the anti-saccade task (B, right diagram)

1991). Indeed, single neuron recordings in monkeys have demonstrated that some SEF neurons have a higher activity on correct anti-saccade trials than on error trials when a reflexive saccade was generated (Schlag-Rey et al. 1997). The fixation hypothesis, therefore, contends that a reduced fixation signal is responsible for the generation of reflexive saccades in the anti-saccade task (Fig. 1A).

In contrast to fixation-related neurons, saccade-related neurons in the SC discharge a high-frequency burst of action potentials for saccades to a certain region of the visual field (their response field), and often exhibit a burst of action potentials when a visual stimulus is presented into this region. While not incompatible with the fixation hypothesis, the "visuomotor" hypothesis (Dorris et al. 1997; Edelman and Keller 1996; Paré and Munoz 1996; Sommer 1994) contends that the ultimate mechanism of short-latency reflexive saccade generation is not reduced fixation activity, but rather a visual signal that passes the saccade threshold of SC saccade-related neurons (Fig. 1A). Indeed, it has been found that errors in the anti-saccade task are associated with a high level of neural activity in SC saccade-related neurons immediately prior to stimulus presentation and a high frequency burst of action potentials in response to stimulus presentation (Everling et al. 1998a; Everling et al. 1999). Based on these findings, Everling et al. (1998a, 1999) have proposed that errors in the anti-saccade task occur when the visual stimulus is presented into the response field of SC saccade-related neurons, which already have a high level of prestimulus activity. In this case, the stimulus- related burst may directly pass the threshold for generating a saccade.

One way to test these two hypotheses is to investigate the effects of reduced neural activity on anti-saccade performance. The fixation hypothesis predicts that subjects will generate more errors because of a reduced suppression signal, whereas the visuomotor hypothesis predicts that subjects will generate fewer errors because of reduced preparatory and visual signals. A reduction in cortical activity can be achieved by the administration of ethanol. At the single channel level, ethanol positively modulates the inhibitory $\gamma$-aminobutyric $\operatorname{acid}_{\mathrm{A}}\left(\mathrm{GABA}_{\mathrm{A}}\right)$ receptor and suppresses $N$-methyl-D-aspartate (NMDA)ergic functions (Davies and Alkana 2001; Wang et al. 1999). The inhibitory effect of ethanol on cortical activity can also be demonstrated at the macro-electrophysiological level with event-related potential (ERP) recordings. One common finding is a decrease in the N1 ERP component in both humans (Krull et al. 1994; Liu et al. 2000) and rodents (Slawecki et al. 2000). Given that there is an assumption within the literature that ethanol causes a reduction in cortical activity, we sought to test the two hypotheses by examining the effects of moderate alcohol consumption on anti-saccade performance in healthy subjects. An increase in the number of direction errors after ethanol would support the fixation hypothesis (Fig. 1B, left diagram), whereas a decrease would support the visuomotor hypothesis (Fig. 1B, right diagram).

\section{Methods}

Subjects

Eight males, aged $24-33$ years (mean \pm SD, 27.5 \pm 3.46 ), were paid for their participation as subjects in this experiment and all gave written, informed consent prior to their inclusion in the study. All subjects had normal or corrected-to-normal vision. None of the subjects reported any neurological or psychiatric disorder, previous head injury, or previous history of alcohol abuse. The procedures in this study were approved by the University Research Ethics Board for Health Sciences Research and have therefore been performed in accordance with the ethical standards laid down in the 1964 Declaration of Helsinki.

Electrophysiological recording procedures

Horizontal eye movements were recorded using an electrooculogram (EOG) in a bipolar montage by placing $\mathrm{Ag}-\mathrm{AgCl}$ skin electrodes at the outer canthi of both eyes. Subjects wore the electrodes for approximately 10 min before the start of the task in order to minimize EOG drift.

To monitor cortical activity, we recorded cerebral potentials from 19 sites of the cranium with nonpolarizable $\mathrm{Ag}-\mathrm{AgCl}$ electrodes with Grass EC2 cream as conductive agent. Electrodes were placed according to the international 10-20 system at Fp1, Fp2, F7, F3, Fz, F4, F8, C3, Cz, C4, T5, P3, Pz, P4, T6, Oz and A1 with $\mathrm{A} 2$, as well as at intermediate sites halfway between frontal and central electrodes (FC3, FCz, FC4). All channels were referred online to a $\mathrm{Cz}$ reference and converted offline algebraically to a linked ear reference. A ground electrode was placed just above the eyebrows in the centre of the forehead. All impedances were maintained below $5 \mathrm{k} \Omega$. Signals were amplified between $0.016 \mathrm{~Hz}$ (time constant $10 \mathrm{~s}$ ) and $70 \mathrm{~Hz}$ using a paperless commercially 
available EEG system (Walter Graphtek, Bad Oldesloe, Germany) and were sampled at $167 \mathrm{~Hz}$.

\section{General procedures}

Each participant completed the study under two conditions, ethanol and placebo, on separate days. Participants expected that ethanol would be consumed in both conditions. The order of the ethanol and placebo conditions was counterbalanced across participants. All testing began at 1300 hours, and participants were asked to consume a light, low-fat meal approximately $2 \mathrm{~h}$ before testing to avoid adverse effects from consuming ethanol on an empty stomach.

In the ethanol condition, participants were served an amount of ethanol (40\% ethyl alcohol by volume) mixed with fruit juice in a $1: 4$ ratio. In the placebo condition, participants received an amount of juice equal to that of liquid volume in the ethanol condition. Drinks were served in cups with lids, and consumed through a straw. A drop of peppermint extract was placed on the lid to mask the smell of ethanol. In the placebo condition, a few drops of ethanol were also added to the lid.

The number of drinks to be consumed by each participant was calculated using the Computerized Blood Alcohol Calculator (CBAC; Addiction Research Foundation, Toronto, Canada 1992), based on the participant's sex, weight, height, and age. Participants were asked to consume a number of drinks estimated to raise blood alcohol concentrations (BACs) in the ethanol condition to $0.08 \%$. The average weight of the eight subjects was $174 \mathrm{lbs}(79.2 \mathrm{~kg})$. They drank on average $4 \mathrm{oz}(118 \mathrm{ml})$ of $40 \%$ alcohol to achieve a BAC level of at least $0.06 \%$. This is equivalent to four standard drinks in Canada. Participants were instructed to consume the drinks within a period of $20 \mathrm{~min}$. Blood alcohol concentrations were first measured 15 min after the 20-min drinking period, then every 15 min until a minimum BAC required for testing was reached. BACs were determined with a breath-measuring device (Alcometer 7410; Draeger, Inc., Durango, CO, USA). Participants were tested twice within each condition. In the ethanol condition, data were collected on both the ascending and descending limbs of the BAC curve. Because to date there has been no examination of anti-saccade performance post-alcohol across the limbs of the BAC curve, we were interested in determining whether there were any differential effects. Data collection in the ethanol condition began upon reaching a $\mathrm{BAC}$ of $0.06 \%$ or greater, and resumed upon reaching a $\mathrm{BAC}$ of less than or equal to $0.06 \%$. In the placebo condition, data were obtained 15 and 30 min after the consumption of the juice.

\section{Behavioural task}

Once participants were ready for testing, they were seated in a comfortable chair in a dimly illuminated room with their arms resting on a table. They were asked to keep their heads upright. In order to reduce artifacts from jaw muscles, no chinrest was used. Two Pentium PCs running CORTEX program (Dr. Robert Desimone, NIMH) were used to control the experiment. Stimuli were presented on a 21 -inch computer monitor placed $80 \mathrm{~cm}$ in front of the subjects. At this distance, the screen subtended $36^{\circ}$ of visual angle. Each trial began with the presentation of a $0.2^{\circ}$ diameter, white fixation point (FP) placed in the centre of the dark monitor screen. After an interval that varied randomly between 1000 $1500 \mathrm{~ms}$, the FP was extinguished. To increase the likelihood of subjects making a direction error, there was then a period of $200 \mathrm{~ms}$ when the screen was blank. This was followed by a white $0.5^{\circ}$ circle presented either $10^{\circ}$ to the left or $10^{\circ}$ to the right of the FP for $500 \mathrm{~ms}$. The left-right presentations were interleaved in a quasirandom order and occurred with equal probability. Subjects were instructed to maintain fixation during the visual fixation and blank periods, and to look away from the stimulus to its mirror location as quickly as possible. Trials were separated by $2000 \mathrm{~ms}$. Subjects performed 400 trials (200 trials per block) on each of the two test days. Each block was separated by a break of at least $15 \mathrm{~min}$ in both conditions. Subjects were required simply to sit quietly during the break.

\section{Data analysis}

In an offline analysis, saccade onsets and direction errors were automatically identified in a time window of $500 \mathrm{~ms}$ prior to and $400 \mathrm{~ms}$ after stimulus presentation. The onset of a saccade could easily be identified in the EOG as the beginning of a brisk movement of the signal away from baseline. Trials with artifacts (e.g. blinks, no eye movement, or large amplitude shifts), anticipations (saccadic reaction times, SRTs $<80 \mathrm{~ms}$ ), or SRTs of more than $400 \mathrm{~ms}$ (indicating that the subject had not correctly perform the task) were automatically excluded from further analysis (Bell et al. 2000; Everling et al. 1998c; Everling et al. 1999). Additionally, all trials were visually inspected for artifacts and the correct identification of the onset of the saccade. Blinks and vertical eye movements were best seen at Fp1 and Fp2 and were excluded from averaging.

The ERPs of the ethanol and placebo condition were averaged separately. We combined trials with left and right stimulus presentations. Findings from preliminary experiments, as well as analysis of findings from the current experiment, demonstrated no significant differences across the two experimental blocks; thus, the two experimental blocks were combined. This increase in the number of trials improved the signal-to-noise ratio. To obtain ipsilateral, midline and contralateral responses in terms of recording site with respect to stimulus location, data from target stimulus presentations to both sides were pooled. For example, to obtain a contralateral response, the ERP recorded at P4, evoked by the left visual field stimulus, was pooled with the ERP recorded at P3, evoked by the right visual field stimulus. The averaging period comprised $100 \mathrm{~ms}$ prior to and $600 \mathrm{~ms}$ after FP offset, i.e. $400 \mathrm{~ms}$ after peripheral stimulus onset. The averaged ERP data were baseline corrected (100 ms prior to FP offset) and filtered digitally with a $30-\mathrm{Hz}$ zero-phase low-pass filter. Grand average waveforms were generated separately for the ethanol and placebo conditions for all subjects.

Mean activity levels were measured before and during the visual stimulation period. As cortical neurons throughout the visual system in primates have response latencies $>50 \mathrm{~ms}$ (Schmolensky et al. 1998), and because Everling et al. (2001) have shown that the activity in the interval from $48 \mathrm{~ms}$ before to $48 \mathrm{~ms}$ after stimulus presentation reflects the cortical activation level immediately before the arrival of the visual signal in the cortex, we measured at the end of the gap period (t1, $48 \mathrm{~ms}$ before to $48 \mathrm{~ms}$ after stimulus presentation). To determine activity levels during the visual stimulation period ( $\mathrm{t} 2$ ), we measured activity levels $54 \mathrm{~ms}$ to $150 \mathrm{~ms}$ after stimulus presentation.

Statistical comparisons were conducted using a repeated measures analysis of variance (ANOVA) for gap-related activity (t1) and stimulus-related activity (t2). The analysis included 12 electrodes (F3, F4, Fz, FC3, FC4, Fz4, C3, C4, Cz, P3, P4, and Pz), with the factors "condition" (placebo or ethanol), "anteriorposterior" (frontal, fronto-central, central, and posterior) and "laterality" (ipsilateral, midline, contralateral). All values are reported as means \pm standard error.

\section{Results}

\section{Blood alcohol concentration}

The BAC achieved on the first block of testing ranged between 0.06 and $0.07 \%$ (mean \pm SE, $0.065 \pm 0.0012 \%$ ), and between 0.043 and $0.06 \%(0.055 \pm 0.007 \%)$ on the second block. 
A

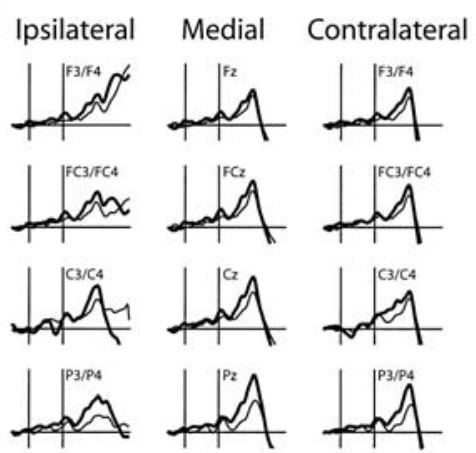

B

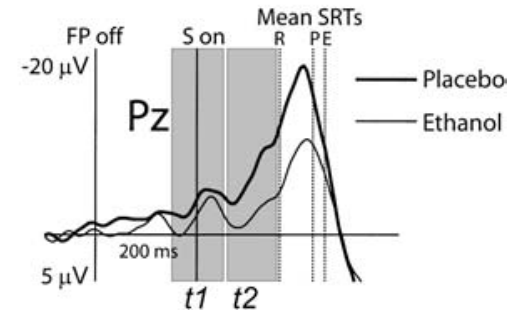

Fig. 2A,B Effects of ethanol (mean blood alcohol concentration $0.06 \%$ ) on event-related potential (ERP) grand averages in the antisaccade task. A Waveforms represent linked ear-references averages from eight normal subjects under placebo and ethanol conditions. The first vertical line indicates the offset of the central fixation point (FP off in $\mathbf{B}$ ); and the second vertical line indicates the onset of the peripheral stimulus ( $S$ on in B). B ERP measurements at $\mathrm{Pz}$. The dashed vertical lines indicate the mean latencies of reflexive saccades $(R)$, correct anti-saccades in the placebo $(P)$ and ethanol $(E)$ conditions. Cortical activity during these two conditions was calculated from $48 \mathrm{~ms}$ before to $48 \mathrm{~ms}$ after stimulus presentation (end of gap period, $t 1$ ) and from $54 \mathrm{~ms}$ to $150 \mathrm{~ms}$ after stimulus presentation (visual activity, $t 2$ )

\section{Event-related potentials}

Figure 2 displays the grand ERP averages in the placebo (thick lines) and ethanol condition (thin lines) in the gap anti-saccade task. The recordings show the same pattern of ERPs that has been described for this task previously (Everling et al. 1998c; Gomez et al. 1996; Spantekow et al. 1999). The disappearance of the fixation point elicited a negative potential at the end of the gap period, the socalled gap-negativity. This negative potential was followed by a positive deflection and then a large negative potential elicited by the appearance of the peripheral stimulus and the motor potential for the saccade.

The effect of ethanol is strongly evident in these recordings: the majority of recording positions showed markedly reduced amplitudes of the ERP in the ethanol condition. A three-way ANOVA with the factors condition (placebo or ethanol), anterior-posterior (frontal, fronto-central, central, and posterior) and laterality (ipsilateral, midline, contralateral) for the gap-related activity showed a significant main effect for condition $\left(F_{(1,7)}=6.2\right.$, $P<0.05$; Fig. $2 \mathrm{~B}, t 1)$. No other main effect or interactions were significant. A similar finding was obtained for the stimulus-related activity. A three-way ANOVA with the same factors as above showed a clear significant effect for
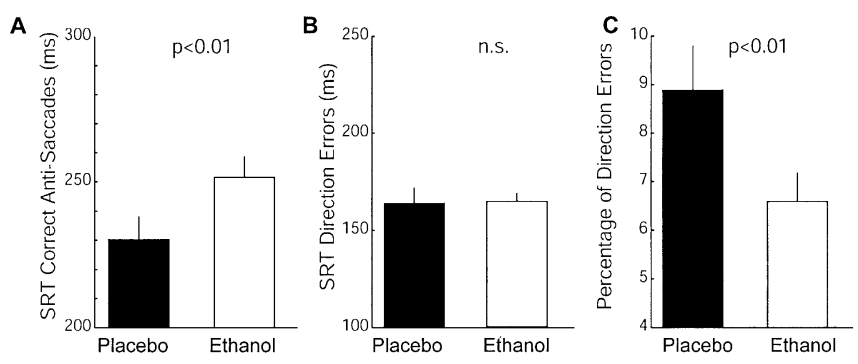

Fig. 3A-C Behavioural data from the placebo and ethanol condition in the anti-saccade task. Means \pm standard errors are shown for saccadic reaction times (SRTs) of correct anti-saccades (A), SRTs of direction errors $(\mathbf{B})$ and percentage of direction errors $(\mathbf{C})$ in the placebo condition (black bars) and ethanol condition (white bars)

condition $\left(F_{(1,7)}=15.1, P<0.0001\right.$; Fig. $\left.2 \mathrm{~B}, t 2\right)$, but no other significant main effects or interactions. These results suggest that ethanol in the anti-saccade task led to general reduction in cortical activity without affecting any specific cortical location.

\section{Behavioural effects}

Figure 3A shows the mean SRTs of correct anti-saccades for the sample of eight subjects in the placebo and ethanol condition. Six of the eight subjects had longer reaction times in the ethanol condition than in the placebo condition. Across subjects, the mean SRTs of correct responses in the placebo and ethanol condition were $229.4 \pm 8 \mathrm{~ms}$ and $251.1 \pm 7 \mathrm{~ms}$, respectively. This difference was significant $\left(t_{(7)}=3.68, P<0.01\right)$. In contrast, the mean SRTs of direction errors were similar for the two conditions (Fig. 3B). The mean SRT of errors was $163.5 \pm 8 \mathrm{~ms}$ in the placebo condition and $164.4 \pm 4 \mathrm{~ms}$ in the ethanol condition $\left(t_{(7)}=0.11, P=0.92\right)$.

Next, we compared the proportion of direction errors in the placebo and ethanol condition (Fig. 3C). We found that seven of the eight subjects made fewer errors in the ethanol condition. The mean percentage of errors dropped from $8.9 \pm 0.9 \%$ in the placebo condition to $6.6 \pm 0.6 \%$ in the ethanol condition $\left(t_{(7)}=3.76, P<0.01\right)$.

Finally, we tested for a possible order effect of condition, and found this to be insignificant.

\section{Discussion}

In the present study, we have investigated the effects of moderate levels of ethanol on anti-saccade performance and ERPs in human subjects. We found that ethanol increases the latency of anti-saccades but not of reflexive responses. Furthermore, subjects generated significantly fewer reflexive saccades in the ethanol condition than in the placebo condition. This is the first study that has also measured the effects of ethanol on ERPs in the antisaccade task. We found a clear reduction in all ERP potentials, indicating a general decrease in cortical 
activity following ethanol intake. These results support the visuomotor hypothesis, which predicts a decrease in reflexive saccades in the anti-saccade task after a decrease in cortical activation.

An increase in the latency of pro-saccades following ethanol has been reported in many studies (Baloh et al. 1979; Blekher et al. 2002; Jantti et al. 1983; Moser et al. 1998; Vassallo and Abel 2002; Wegner and Fahle 1999). The prolonged latencies of pro-saccades have been attributed to an effect of ethanol on processing in the cortical parietal and frontal eye fields and the SC (Baloh et al. 1979; Jantti et al. 1983). In contrast to the clear effects of ethanol on pro-saccades, there is a relative paucity of studies on anti-saccades post-alcohol. Further, the few studies that have investigated the effects on antisaccades have yielded conflicting results. For example, Crevits et al. (2000) found a slightly higher error rate and longer latencies in the anti-saccade task in alcoholintoxicated subjects with head trauma than in sober subjects with head trauma. It should be noted that the findings from the study by Crevits and colleagues are not widely generalizable because the subjects were extremely intoxicated as well as victims of traumatic brain injury. Blekher et al. (2002) also found an increase in antisaccade latency after ethanol, as well as in anti-saccades that were more hypermetric. Recently, Vassallo and Abel (2002) reported a slight decrease in the error rate after ethanol intake; however, they attributed this decrease to a learning effect because their subjects always performed the non-alcohol control trials first. We randomized the order of the placebo and ethanol conditions across subjects in our study, and can therefore rule out such an effect. It should also be noted that the subjects in the previous studies were only tested on 30-40 anti-saccade trials, whereas our subjects completed 400 trials in each condition. A large number of trials clearly make it more likely to detect small changes in error rate. Moreover, these previous studies used a step design, i.e. the central fixation point disappeared at the same time as the peripheral stimulus was presented. It has been shown that, by introducing a time interval between fixation and peripheral stimulus onset, an increase in the number of errors occurs in an anti-saccade task (Bell et al. 2000; Fischer and Weber 1997). This design is therefore more suitable for investigating error-related processes.

Evoked-potential recordings in awake behaving monkeys have shown that ethanol reduces visual responses in a variety of brain areas, including the lateral geniculate nucleus, the striate cortex, and the inferotemporal cortex (Fuster et al. 1982). A reduction of the P1 and N1 visual ERP components has been found in many studies in human subjects (for review see Jääskeläinen et al. 1996). This reduction has been attributed to an increase in GABA-ergic and a decrease in NMDA-ergic functions (Davies and Alkana 2001; Wang et al. 1999). Our study confirms the suppressive effect of alcohol on ERPs. Previously, it had been hypothesized that ethanol at low doses has a greater effect on the posterior parietal cortex than the FEF (Vassallo and Abel 2002). Our data do not reveal any clear region-specific effects of ethanol on ERPs associated with the gap anti-saccade task, and therefore suggest that ethanol attenuates neural processing in a rather generalized way.

According to the visuomotor hypothesis reflexive saccades are elicited in the gap anti-saccade task when the visual signal impinges on saccade-related neurons in the SC that already have a high prestimulus activity at the end of the gap period. Our ERP data show that ethanol reduces the amplitude of the gap negativity, which would be consistent with a decreased cortical activity at the end of the gap period. Our data also demonstrate reduced ERPs in response to the visual stimulus, indicating reduced cortical visual activity in the ethanol condition. We therefore cannot ascertain from our data whether a reduced prestimulus activity, a reduced visual signal or a combination of both is responsible for the reduced error rates in the ethanol condition.

A reduction in the level of motor preparation after ethanol consumption would not only explain the lower rate of direction errors but also the longer latencies of correct anti-saccades. Single neuron recordings have demonstrated that the level of prestimulus activity of saccade-related neurons in the SC ipsilateral to the stimulus (i.e. contralateral to the movement) is negatively correlated with the reaction time of the subsequent antisaccade (Everling et al. 1999). A reduction in motor preparation would therefore reduce the number of errors but also increase reaction times of correct anti-saccades.

Although the visuomotor hypothesis would predict that a weaker visual signal would be less likely to trigger a reflexive saccade, there is currently no evidence that low stimulus luminances reduce reflexive saccades in the antisaccade task. In fact, two studies that investigated the effects of different stimulus luminance on anti-saccade performance found an increase in the error rate at scotopic and mesopic (between foveal threshold to $1 \log$ unit above foveal threshold) stimulus luminance (Doma and Hallett 1988, 1989). The finding of an increase in error rate on anti-saccade performance under scotopic and mesopic conditions, however, may simply reflect perceptual difficulties in localizing the stimulus since high error rates were also found for pro-saccades under scotopic conditions (Doma and Hallett 1988). The authors hypothesized that two visual signals, the first from cones and the second a delayed signal from rods, are generated under mesopic luminances. Doma and Hallett speculated that the two visual signals would make it difficult to ignore these requests to foveate. There are no data to date, however, that support this hypothesis. Indeed, functional magnetic resonance imaging studies have shown that low stimulus luminances are associated with a reduced bloodoxygenation level-dependent (BOLD) signal in the striate cortex (Goodyear and Menon 1998; Mohamed et al. 2002). Further studies that systematically test the relationship between stimulus luminance and error rates in the anti-saccade task are needed to test whether an increase in stimulus luminance leads to higher error rates when the level of motor preparation is kept constant. 
In summary, our data show that ethanol reduces the amplitude of evoked potentials and reduces the number of errors in the gap anti-saccade task. Taken together, our findings support the visuomotor hypothesis, which contends that direction errors occur in the anti-saccade task when the impinging visual signal in the SC directly passes the saccade threshold.

Acknowledgements This work was supported by grants from the Natural Science Engineering Research Council of Canada to B.T. and S.E. S.E. is a National Alliance for Research on Schizophrenia and Depression Young Investigator and a Canadian Institutes of Health Research New Investigator. We are especially grateful to Hans Flohr for lending us the EEG system and to Astrid Matthews for her help in setting it up.

\section{References}

Baloh RW, Sharma S, Moskowitz H, Griffith R (1979) Effect of alcohol and marijuana on eye movements. Aviat Space Environ Med 50:18-23

Bell AH, Everling S, Munoz DP (2000) Influence of stimulus eccentricity and direction on characteristics of pro- and antisaccades in non-human primates. J Neurophysiol 84:2595-2604

Blekher T, Beard JD, O'Connor S, Orr WE, Ramchandani VA, Miller K, Yee RD, Li TK (2002) Response of saccadic eye movements to alcohol in African American and non-Hispanic white college students. Alcohol Clin Exp Res 26:232-238

Crevits L, Hanse MC, Tummers P, Van Maele G (2000) Antisaccades and remembered saccades in mild traumatic brain injury. J Neurol 247:179-182

Davies DL, Alkana RL (2001) Ethanol enhances $\mathrm{GABA}_{\mathrm{A}}$ receptor function in short sleep and long sleep mouse brain membranes. Alcohol Clin Exp Res 25:478-483

Doma H, Hallett PE (1988) Dependence of saccadic eye-movements on stimulus luminance, and an effect of task. Vision Res 28:915-924

Doma H, Hallett PE (1989) Variable contributions of rods and cones to saccadic eye-movement latency in a non-foveating task. Vision Res 29:563-577

Dorris MC, Paré M, Munoz DP (1997) Neuronal activity in monkey superior colliculus related to the initiation of saccadic eye movements. J Neurosci 17:8566-8579

Edelman JA, Keller EL (1996) Activity of visuomotor neurons in the superior colliculus accompanying express saccades. J Neurophysiol 76:908-926

Everling S, Fischer B (1998) The antisaccade: a review of basic research and clinical studies. Neuropsychologia 36:885-899

Everling S, Dorris MC, Munoz DP (1998a) Reflex suppression in the antisaccade task is dependent on prestimulus neural processes. J Neurophysiol 80:1584-1589

Everling S, Paré M, Dorris MC, Munoz DP (1998b) Comparison of the discharge characteristics of brain stem omnipause neurons and superior colliculus fixation neurons in monkey: implications for control of fixation and saccade behaviour. J Neurophysiol 79:511-528

Everling S, Spantekow A, Krappmann P, Flohr H (1998c) Eventrelated potentials associated with correct and incorrect responses in a cued antisaccade task. Exp Brain Res 118:27-34

Everling S, Dorris MC, Klein RM, Munoz DP (1999) Role of primate superior colliculus in preparation and execution of antisaccades and pro-saccades. J Neurosci 19:2740-2754

Everling S, Matthews, A, Flohr, H (2001) Prestimulus cortical potentials predict the performance in a saccadic distracter paradigm. Clin Neurophysiol 112:1088-1095
Fischer B, Weber H (1997) Effects of stimulus conditions on the performance of antisaccades in man. Exp Brain Res 116:191200

Fuster JM, Willey TJ, Riley DM, Ashford JW (1982) Effects of ethanol on visual evoked responses in monkeys performing a memory task. Electroencephalogr Clin Neurophysiol 53:62133

Gaymard B, Ploner CJ, Rivaud S, Vermersch AI, Pierrot-Deseilligny C (1998) Cortical control of saccades. Exp Brain Res 123:159-163

Gomez C, Atienza M, Gomez GJ, Vazquez M (1996) Response latencies and event-related potentials during the gap paradigm using saccadic responses in human subjects. Int J Psychophysiol 23:91-99

Goodyear BG, Menon RS (1998) Effect of luminance contrast on BOLD fMRI response in human primary visual areas. J Neurophysiol 79:2204-2207

Guitton D, Buchtel HA, Douglas RM (1985) Frontal lobe lesions in man cause difficulties in suppressing reflexive glances and in generating goal-directed saccades. Exp Brain Res 58:455-472

Hallett PE (1978) Primary and secondary saccades to goals defined by instructions. Vision Res 18:1279-1296

Hallett PE, Adams BD (1980) The predictability of saccadic latency in a novel voluntary oculomotor task. Vision Res 20:329-339

Hess WR, Burgi S, Bucher V (1946) Motor function of tectal and tegmental area. Monatsschr Psychiatr Neurol 112:1-52

Jääskeläinen IP, Näätänen R, Sillanaukee P (1996) Effect of acute ethanol on auditory and visual event-related potentials: a review and reinterpretation. Biol Psychiatry 40:284-291

Jantti V, Lang AH, Keskinen E, Lehtinen I, Pakkanen A (1983) Acute effects of intravenously given alcohol on saccadic eye movements and subjective evaluations of intoxication. Psychopharmacology (Berl) 79:251-255

Krull KR, Smith LT, Parsons OA (1994) Simple reaction time event-related potentials: effects of alcohol and diazepam. Prog Neuropsychopharmacol Biol Psychiatry 18:1247-1260

Liu Y, Higuchi S, Motohashi Y (2000) Time-of-day effects of ethanol consumption on EEG topography and cognitive eventrelated potential in adult males. J Physiol Anthropol Appl Human Sci 19:249-254

Mohamed FB, Pinus AB, Faro SH, Patel D, Tracey JI (2002) BOLD fMRI of the visual cortex: quantitative responses measured with a graded stimulus at 1.5 Tesla. J Magn Reson Imaging 16:128136

Moser A, Heide W, Kompf D (1998) The effect of oral ethanol consumption on eye movements in healthy volunteers. J Neurol 245:542-550

Munoz DP, Wurtz RH (1992) Role of rostral superior colliculus in active visual fixation and execution of express saccades. J Neurophysiol 67:1000-1002

Paré M, Munoz DP (1996) Saccadic reaction time in the monkey: advanced preparation of oculomotor programs is primarily responsible for express saccade occurrence. J Neurophsyiol 76:3666-3681

Pierrot-Deseilligny C, Rivaud S, Gaymard B, Agid Y (1991) Cortical control of reflexive visually-guided saccades. Brain 114:1473-1485

Pierrot-Deseilligny C, Israel I, Berthoz A, Rivaud S, Gaymard B (1993) Role of the different frontal lobe areas in the control of the horizontal component of memory-guided saccades in man. Exp Brain Res 95:166-171

Schlag-Rey M, Amador N, Sanchez H, Schlag J (1997) Antisaccade performance predicted by neuronal activity in the supplementary eye field. Nature 390:398-401

Schmolensky MT, Wang Y, Hanes DP, Thompson KG, Leutgeb S, Schall JD, Leventhal AG (1998) Signal timing across the macaque visual system. J Neurophysiol 79:3272-3278

Slawecki CJ, Walpole T, Purdy RH, Ehlers CL (2000) Comparison of the neurophysiological effects of allopregnanolone and ethanol in rats. Psychopharmacology (Berl) 149:351-359 
Sommer MA (1994) Express saccades elicited during visual scan in the monkey. Vision Res 34:2023-2038

Spantekow A, Krappmann P, Everling S, Flohr H (1999) Eventrelated potentials and saccadic reaction times: effects of fixation point offset or change. Exp Brain Res 127:291-297

Vassallo S, Abel LA (2002) Ethanol effects on volitional versus reflexive saccades. Clin Experiment Ophthalmol 30:208-212

Wang MY, Rampil IJ, Kendig JJ (1999) Ethanol directly depresses AMPA and NMDA glutamate currents in spinal cord motor neurons independent of actions on $\mathrm{GABA}_{\mathrm{A}}$ or glycine receptors. J Pharmacol Exp Ther 290:362-367

Wegner AJ, Fahle M (1999) Alcohol and visually guided saccades: gap effect and predictability of target location. Psychopharmacology (Berl) 146:24-32

Wurtz RH, Goldberg ME (1989) The neurobiology of saccadic eye movements. Elsevier, Amsterdam 